\title{
Investigation on the flexural properties of nanofillers loading on the Jute/Carbon/PLA nanocomposites
}

\begin{abstract}
Presence of fibers and fillers in a composite can be an efficient way to arrest crack either at macro or micro levels. In this work, woven jute and carbon fibers were arranged alternately in PLA (Polylactic Acid) nanocomposite. Graphene or nanoclay was embedded into PLA matrix to make polymer nanocomposite. Fiber reinforced polymer nanocomposites were prepared by varying the concentration of graphene or nanoclay in the PLA matrix and alternate woven jute/carbon fibers was then bind with the PLA nanocomposite. Influence of graphene or nanoclay concentration and presence of woven fibres in the composite was quantified by flexural analysis. Flexural strength and flexural modulus were found to increase at $3 \mathrm{wt} \%$ of nanofiller concentration for both graphene/jute/PLA and nanoclay/jute/PLA nanocomposites with increment up to $37 \%$ and $31 \%$, respectively. FTIR was used to determine the interaction between PLA and nanofillers. Morphology observation by Scanning Electron Microscopy (SEM) was done to investigate the fractured surface of the hybrid jute/carbon fibres reinforced PLA nanocomposite.
\end{abstract}

Keyword: Carbon fibre; Flexural graphene; Jute fibre; Nanoclay; Polylactic acid 